\title{
Development of a RF-driven ion source for the ITER NBI system
}

\author{
A. Staebler*, U. Fantz, P. Franzen, M. Berger, S. Christ-Koch, H. D. Falter, M. Froeschle, \\ R. Gutser, B. Heinemann, D. Holtum, W. Kraus, C. Martens, P. McNeely, R. Nocentini, \\ S. Obermayer, R. Riedl, E. Speth, D. Wünderlich
}

Max-Planck-Institut für Plasmaphysik, EURATOM Association, 85748 Garching, Germany

* Corresponding author. Tel. +49 893299 1617, email: albrecht.staebler@ipp.mpg.de

\begin{abstract}
Extensive R\&D work on RF-driven negative hydrogen ion sources carried out at IPP Garching led to the decision of ITER to select this type of source as the new reference source for the ITER NBI system. The principle suitability of the RF source has been demonstrated in a small scale, short pulse length experiment: accelerated current densities, co-extracted electron currents at a source operation pressure, all well inside the range of the ITER requirements have been achieved simultaneously. In subsequent experiments, pulse lengths up to $1 \mathrm{~h}$ and the possibility of modularly extending the source to ITER source dimensions were demonstrated. The results achieved at the various IPP test beds, the lessons learnt during optimising the source for negative ion production and extraction as well as the problems still to be solved are summarized. As the next step in support of the NBI development for ITER, IPP plans to build a new test facility for beam extraction from a source of half the size for ITER.
\end{abstract}

Keywords: Neutral Beam Injection, Negative Ion Source, RF Source

\section{Introduction}

Neutral beam injection (NBI) heating for the ITER tokamak foresees [1] $1 \mathrm{MeV}$ deuterium beams from two injectors with a total power of $33 \mathrm{MW}$ and a pulse length of $1 \mathrm{~h}$. This is to be achieved by extracting and accelerating negative deuterium ions from a plasma source of roughly $1.8 \mathrm{~m} \times 0.9 \mathrm{~m}$ with a net extraction area of $0.2 \mathrm{~m}^{2}$. At a source filling pressure of $0.3 \mathrm{~Pa}$, the accelerated current density (i.e. the current measured on a calorimeter after acceleration) has to be $\mathrm{j}_{\mathrm{D}^{-}}=200 \mathrm{~A} / \mathrm{m}^{2}$ with $\mathrm{j}_{\mathrm{e}^{-}} / \mathrm{j}_{\mathrm{D}^{-}}<1, \mathrm{j}_{\mathrm{e}}-$ being the current density of the co-extracted electrons.

In 2007, ITER has selected a RF-driven plasma source as being the reference source for NBI - a decision mainly based on the related R\&D work carried at IPP Garching [2-5]. RF sources, when compared to a filamented arc source, have fewer parts and are in 
principal maintenance free, due to the absence of filaments. This is of particular importance for ITER where remote maintenance is required in a radioactive environment. The status achieved with the RF source and future steps to be undertaken towards an ITER source will be summarized in the paper.

\section{Setup of the IPP RF source}

The principle setup of an IPP RF source is shown in Fig. 1 [3]: RF power $\left(f_{R F}=1 \mathrm{MHz}\right)$ is coupled into a cylindrical driver. A Faraday shield protects its ceramic wall. The plasma created there expands into the main source chamber. A filter field prevents hot electrons to come close to the extraction area where they could destroy negative ions. Negative ions are mainly produced on surfaces covered with a thin Caesium layer [6]. Small amounts of Cs $(\sim 10 \mathrm{mg} / \mathrm{h})$ are therefore fed into the source. Due to the short distance negative ions can cover without being destroyed (a few $\mathrm{cm}[6]$ ) only those ions created on the plasma grid (PG) are available for extraction. Beam extraction and acceleration is done by a threegrid system. Typical voltage levels of extraction grid (EG) and grounded grid (GG) are given in Fig. 1. Magnets inserted into the EG deflect the co-extracted electrons onto this grid in order to prevent their acceleration to full voltage. The PG can be biased against the source body by some ten volts. The co-called bias plate close to the PG provides a further possibility to control source potentials.

\section{Experimental results from test bed operation}

\subsection{BATMAN operation}

BATMAN (Bavarian test machine for negative ions) is a test bed where power supplies, pumping capability and cooling restrict source operation (source height: $0.6 \mathrm{~m}$, width: 0.3 m) to rather small extraction areas $\left(\sim 70 \mathrm{~cm}^{2}\right)$ and short pulse lengths $(\leq 4 \mathrm{~s})$. Under these conditions, three of the ITER source requirements have been demonstrated simultaneously: Accelerated current densities above $300 \mathrm{~A} / \mathrm{m}^{2}$ at $\mathrm{je}_{\mathrm{e}^{-}} / \mathrm{j}_{\mathrm{H}^{-}}<0.5$ in hydrogen 
and above $200 \mathrm{~A} / \mathrm{cm}^{2}$ at $\mathrm{je}_{\mathrm{e}}-/ \mathrm{j}_{\mathrm{D}^{-}}<1$ in deuterium, both at a source filling pressure around $0.3 \mathrm{~Pa}[5]$.

In order to achieve these results, many lessons had to be learnt and additional observations were made that were important for the next steps towards the ITER source. Very early, it turned out that "Cs conditioning", that is Cs evaporation into the source and the different measures to control its distribution on the inner surfaces, is a necessary, but tedious and difficult process [3]. Initial conditioning takes several days (see Fig. 2) and some shots are needed every day to recover the former performance. The determination of the Cs content inside the plasma by optical emission spectroscopy [7] turned out to be a very useful tool for optimising this conditioning. "Good Cs conditions" result in a rather low co-extracted electron content at high ion current. The value of $\mathrm{j}_{\mathrm{e}}-\mathrm{j}_{\mathrm{H}^{-}}$can be further improved by carefully adjusting the strength of the filter field and optimising the bias voltage of PG. A bias voltage close to the floating potential of the PG results in optimum performance [4]. The extractable current density could be significantly increased by introducing chamfers to the plasma grid holes on the plasma side [3]. Hereby, the surface where $\mathrm{H}^{-}$ions are created is increased and, in addition, the starting angles of these ions are more favourable for extraction [5]. The optimum performance of the source is already achieved at temperatures of the plasma grid of around $150^{\circ} \mathrm{C}$ [3], somewhat lower than the values reported from arc sources [8].

Two grid stacks with different diameters of the extraction apertures (LAG $\phi=8 \mathrm{~mm}$, CEA $\phi=14 \mathrm{~mm}$, the reference value for ITER) have been used at BATMAN with no significant difference in the achievable ion current density (Fig. 2). This is in contrast to results reported from single hole extraction experiments [9] but is reproduced in simulation calculations [10]. 
When comparing hydrogen with deuterium operation no big difference is found with respect to source efficiency [3]. Deuterium, however, requires a somewhat higher extraction voltage and, more importantly, the co-extracted electron current is significantly higher as in hydrogen under the same conditions. A stronger filter field is required to reduce the electron current. During the source conditioning phase performance in deuterium operation was often limited by the high power load onto the extraction grid from the co-extracted electrons dumped onto this grid.

The set of diagnostics used on the IPP test beds was mainly developed at BATMAN: Optical emission spectroscopy to determine line integrated plasma parameters, the already mentioned Cs monitor, beam spectroscopy, i.e. Doppler shifted $\mathrm{H}_{\alpha}$ from beam neutrals, Langmuir probes for local plasma parameters, laser detachment and cavity ringdown diagnostics to determine the $\mathrm{H}^{-}$density are described in some detail in refs $[5,11]$. An overview of the various codes developed to simulate source plasma, Cs transport, effects of externally applied potentials, $\mathrm{H}^{-}$extraction, beam formation and others is given in refs $[12,13]$.

Advancing from a BATMAN type ion source towards the ITER source requires significant steps in two directions: (i) The pulse length has to be extended from $4 \mathrm{~s}$ to $1 \mathrm{~h}$; the MANITU test bed is devoted to this task. (ii) The size of the source has to be increased in order to cope with an extraction area of $0.2 \mathrm{~m}^{2}$; the RADI test bed is a first step to demonstrate the concept of modular extension of the IPP RF source. ELISE, a test bed planned to be built in the near future will combine both, long pulses from a large source of half the size of the ITER source.

\subsection{MANITU operation}

MANITU (Multi ampere negative ion test unit) is a test bed equipped with power supplies as well as cooling and pumping capabilities that allow essentially cw operation [14]. The 
ion source dimensions correspond to those of the BATMAN source except for a larger extraction area: $200 \mathrm{~cm}^{2}$. After a process of continuous system improvements operation with a pulse length of up to $1 \mathrm{~h}$ at stationary ion and electron currents [14] was achieved. Pulses of several hundred seconds in hydrogen with $\mathrm{j}_{\text {ion }} \geq 200 \mathrm{~A} / \mathrm{m}^{2}$ and, simultaneously, $\mathrm{j}_{\mathrm{e}}-/ \mathrm{j}_{\text {ion }}<1$ are now routinely available. An example is shown in Fig. 3. (Note: $\mathrm{j}_{\text {ion }}$ is the electrically measured current density of the extracted ions; typically $70-80 \%$ of this current is accelerated onto the calorimeter [3].)

The main problems to be solved prior to obtaining these results were: (i) Hardening of the RF circuits to make them compatible with reliable long pulses of a RF power of about 100 $\mathrm{kW}$. Modifications on various components of the RF circuits have been done to improve cooling and an SF6 insulation of the RF antenna around the driver was recently installed. Antenna insulation, however, is not an issue for the ITER source since there, the whole source is inside the vacuum. (ii) Achieving and maintaining good Cs conditions for long pulses. In this respect, temperature control of all inner surfaces of the source is an important tool. This is achieved by using tempered water for all source walls and forced airflow through the plasma grid for cooling/heating during and in-between pulses in addition to actively cooling of the Faraday shield [15]. Wall temperatures in the range of $40^{\circ} \mathrm{C}$ help to avoid Cs sinks that may occur on cold surfaces and then provide the danger of becoming sources of sudden, uncontrolled Cs release during a pulse. However, even after optimising the temperature control, a rise of the electron current on a $150 \mathrm{~s}$ time scale was still present. This could be finally overcome by coating of the Faraday shield and of (almost) all inner surfaces with Molybdenum [15]. Prior to this coating thin $\mathrm{Cu}$ layers were found inside the source after some time of operation and $\mathrm{Cu}$ was observed spectroscopically in the source plasma. $\mathrm{Cu}$ can be sputtered from uncoated surfaces, mainly from Faraday shield and grids, due to high energy plasma and back-streaming ions. This $\mathrm{Cu}$ is suspected to poison the Cs layers during long pulses leading to a loss of 
performance. Fig. 4 shows the significant improvement in performance observed in long pulse operation after Mo-coating of the source.

Deuterium operation of MANITU was so far restricted to a few operational days, which is not sufficient to test the maximum performance. High $\mathrm{j}_{\text {ion }}$ close to $200 \mathrm{~A} / \mathrm{m}^{2}$ with $\mathrm{j}_{\mathrm{e}}-\mathrm{j}_{\text {ion }}<$ 1 were, nevertheless, obtained for up to about $100 \mathrm{~s}$. A further rise of $\mathrm{P}_{\mathrm{RF}}$ to increase the extracted current resulted into a too strong rise of the co-extracted electron current (Fig. 5). Further optimisation in longer deuterium campaigns is required to tackle this problem.

\subsection{RADI operation}

The concept of extending the dimensions of the RF source to those required for an ITER source consists of increasing the size of the main source body and adding additional drivers to the back plate. Such a plasma source of roughly half the size of the ITER source with four drivers but no ion extraction is being operated at the RADI test facility [16]. A "dummy plasma grid" of this source allows simulating the vacuum conductance of a grid system. In addition, this "grid" can be biased against the source body and it can be fed with a so-called PG current of up to $5 \mathrm{kA}$ to create a magnetic filter field similarly to that foreseen in the ITER source. The RADI source allows Cs seeding and is operated in deuterium.

The main purposes of RADI are (i) to demonstrate the concept of modular source extension, (ii) to test the ITER-relevant RF circuits: two horizontally neighbouring drivers are connected in serious to one RF generator, (iii) to study plasma uniformity and as far as possible the $\mathrm{H}^{-}$uniformity dependent on the strength of the filter field and on the amount of RF power coupled to the different drivers, all this without and with Cs seeding.

Experiments have been performed so far without Cs seeding of the source. After solving some initial problems with the RF circuits, operation with $\mathrm{P}_{\mathrm{RF}}$ above $100 \mathrm{~kW}$ per generator is now routinely possible. Without filter field, a rather uniform plasma in front 
of the extraction area is observed provided all drivers are fed with the same power. As shown in Fig. 6, scans of $\mathrm{P}_{\mathrm{RF}}$ in the bottom drivers with constant power in the top drivers demonstrate the possibility to modify the plasma intensity in vertical direction. This may help to compensate for possible vertical inhomogeneities coming, for example, from plasma drifts perpendicular to the filter field. The lower intensity with PG current reflects the lower electron temperature in the presence of the filter field [5]. In how far these plasma modifications will influence the negative ion density close to the extraction can only be studied after the planned Cs seeding into the source.

\section{ELISE - a test bed for beam extraction from a large source}

Even though the plasma only operation of the large RADI source is an important step in the development process of the RF source for ITER, the ultimate goal of creating a negative ion beam that is uniform over the large extraction area at the required current density and a sufficiently low co-extracted electron content can be demonstrated only by beam extraction from such a source. To achieve this goal is the main purpose of the ELISE (Extraction from a Large Ion Source Experiment) test facility [17] planned to be built at IPP. The design of various ELISE components strongly benefits from the operational experience obtained during many years of test bed operation [18]. The main parameters of ELISE are given in Table 1.

ELISE is designed to make use as much as possible of existing IPP hardware, including that from MANITU and RADI. Many of the design solutions for ion source and grids are being transferred to the design of the ITER source [19]. ELISE is seen as an important step between small scale extraction experiments and the full size ITER beam source that contributes significantly to the R\&D activities for the ITER NBI system, carried out at RFX, Padova. A detailed description of ELISE is presented during this conference [17].

\section{Summary}


The main achievements of the R\&D work on the RF-driven negative ion source for the NBI on ITER can be summarized as follows: Accelerated current densities, co-extracted electron currents and source filling pressures, all well inside the range of the ITER requirements have been achieved simultaneously in a small scale, short pulse length experiment. Stable beam pulses for up to $1 \mathrm{~h}$ and reliable plasma operation of a large source at relevant RF power levels were demonstrated in subsequent experiments. These results together with the physics insights obtained from detailed diagnostic and modelling efforts were used as the basis to design a plasma source of half the ITER source size with negative ion extraction. The main aim of this experiment is to demonstrate the production of a large, uniform negative ion beam with ITER relevant parameters, suitable to be accelerated up to $1 \mathrm{MeV}$. Here, a reliable control of the Cs inside the source and sufficient electron suppression at high $\mathrm{D}^{-}$currents will be the major challenges. Tackling these problems in the near future will be an important contribution to the R\&D activities for the ITER NBI system.

\section{Acknowledgements}

Most of the work reported here was performed in the framework of various contracts with EFDA (European Fusion Development Agreement). This continuous support over many years is gratefully acknowledged. 


\section{References}

[1] T. Inoue et al., Fusion Eng. Des. 55 (2001) 291-301

[2] E. Speth and NBI-Team, Plasma Sci. Technol. 6 (2004) 2135-2140

[3] E. Speth et al., Nucl. Fusion 46 (2006) S220-S238

[4] P. Franzen et al., Nucl. Fusion 47 (2007) 264-270

[5] U. Fantz et al., Plasma Phys. Control. Fusion 49 (2007) B563-B580

[6] J. Pamela, Plasma Phys. Control. Fusion 37 (1995) A325-A336

[7] U. Fantz et al., Fusion Eng. Des. 74 (2005) 299-303

[8] R. Trainham et al., Rev. Sci. Instrum. 69 (1998) 926-928

[9] L. Svensson, private communication

[10] R. Gutser, to be published

[11] U. Fantz et al., Rev. Sci. Instrum. 77 (2006) 03A516

[12] D. Wünderlich et al., 34th EPS Conf. Plasma Phys. (2007) ECA Vol. 31F, P-1.149

[13] D. Wünderlich et al., 35th EPS Conf. Plasma Phys. (2008) ECA Vol. 32, P-2.102

[14] W. Kraus et al., Rev. Sci. Instrum. 79 (2008) 02C108

[15] W. Kraus et al., 35th EPS Conf. Plasma Phys. (2008) ECA Vol. 32, P-1.103

[16] P. Franzen et al., Fusion Eng. Des. 82 (2007) 407-423

[17] B. Heinemann et al., this conference, to be published in Fusion Eng. Des.

[18] P. Franzen et al., Proc. 1st NIBS 2008 (Aix-en-Provence, France), to be published

[19] D. Marcuzzi et al., this conference, to be published in Fusion Eng. Des. 


\begin{tabular}{|l|l|}
\hline Isotope & $\mathrm{H}, \mathrm{D}$ (limited) \\
\hline Extraction area & $1000 \mathrm{~cm}^{2}$ \\
\hline Source size (h x w) & $1.0 \times 0.86 \mathrm{~cm}^{2}$ \\
\hline $\mathrm{U}_{\mathrm{HV}}$ & $<60 \mathrm{kV}$ \\
\hline $\mathrm{U}_{\mathrm{ex}}$ & $\sim 12 \mathrm{kV}$ \\
\hline $\mathrm{P}_{\mathrm{RF}}$ & $2 \times 180 \mathrm{~kW}$ \\
\hline Pulse length & \\
Plasma & $3600 \mathrm{~s}$ \\
Extraction & $10 \mathrm{~s}$ every $160 \mathrm{~s}$ \\
\hline
\end{tabular}

Table 1: Parameters of ELISE 


\section{Figure Captions}

Fig. 1. Schematic view of the IPP RF source, the extraction region is shown in detail.

Fig. 2. Comparison of the performance for two grid systems, LAG: $\phi=8 \mathrm{~mm}$, CEA: $\phi=14$ $\mathrm{mm}$. Shown are data obtained during the two experimental campaigns. Obviously, high performance is obtained only after many days of operation.

Fig. 3. Extracted ion and electron currents of a long pulse in hydrogen operation. The maximum ion current correspond to $j_{\text {ion }}=230 \mathrm{~A} / \mathrm{m}^{2}$.

Fig. 4. Performance obtained in MANITU prior to and after Mo-coating. High ion current densities at low electron to ion ratio for long pulses were only obtained after Mo-coating.

Fig. 5. Extracted ion and electron currents (4 A correspond to $200 \mathrm{~A} / \mathrm{m}^{2}$ ) obtained during a short period of deuterium operation. Due to insufficient Cs conditioning, the electron current becomes high and unstable for the higher RF power.

Fig. 6. Line-integrate $\mathrm{H}_{\beta}$-light from horizontal viewing lines close to the extraction region in RADI ( $t$ : line along top part of source, $c$ : central line, $b$ : bottom line). The data were taken without $\left(\mathrm{I}_{\mathrm{PG}}=0\right)$ and with filter field $\left(\mathrm{I}_{\mathrm{PG}}=5 \mathrm{kA}\right)$ for constant $\mathrm{RF}$ power $(80 \mathrm{~kW})$ in the top drivers and a power scan $(40-100 \mathrm{~kW})$ in the bottom drivers. The lower intensity with filter field reflects the lower electron temperature in this case. 


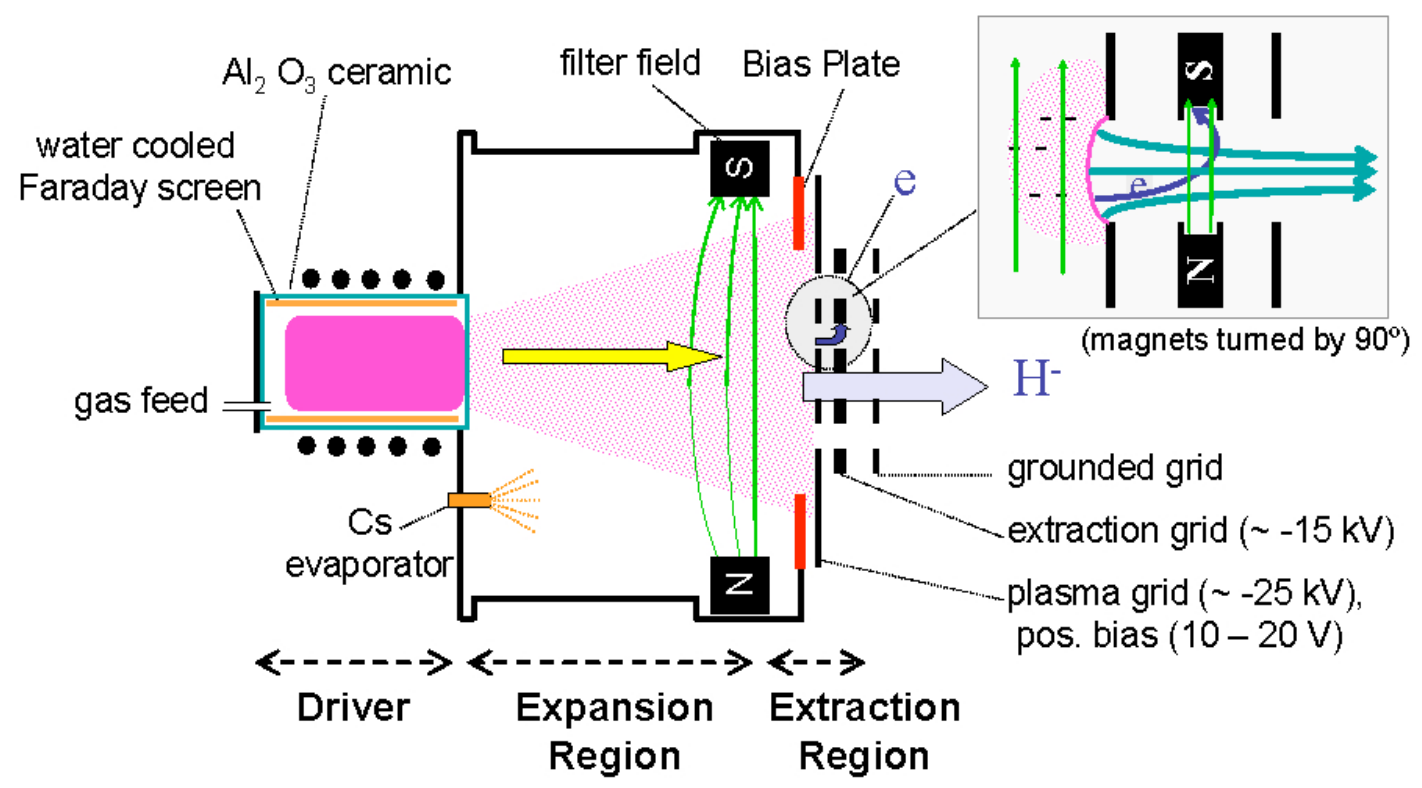

Fig. 1. Schematic view of the IPP RF source, the extraction region is shown in detail.

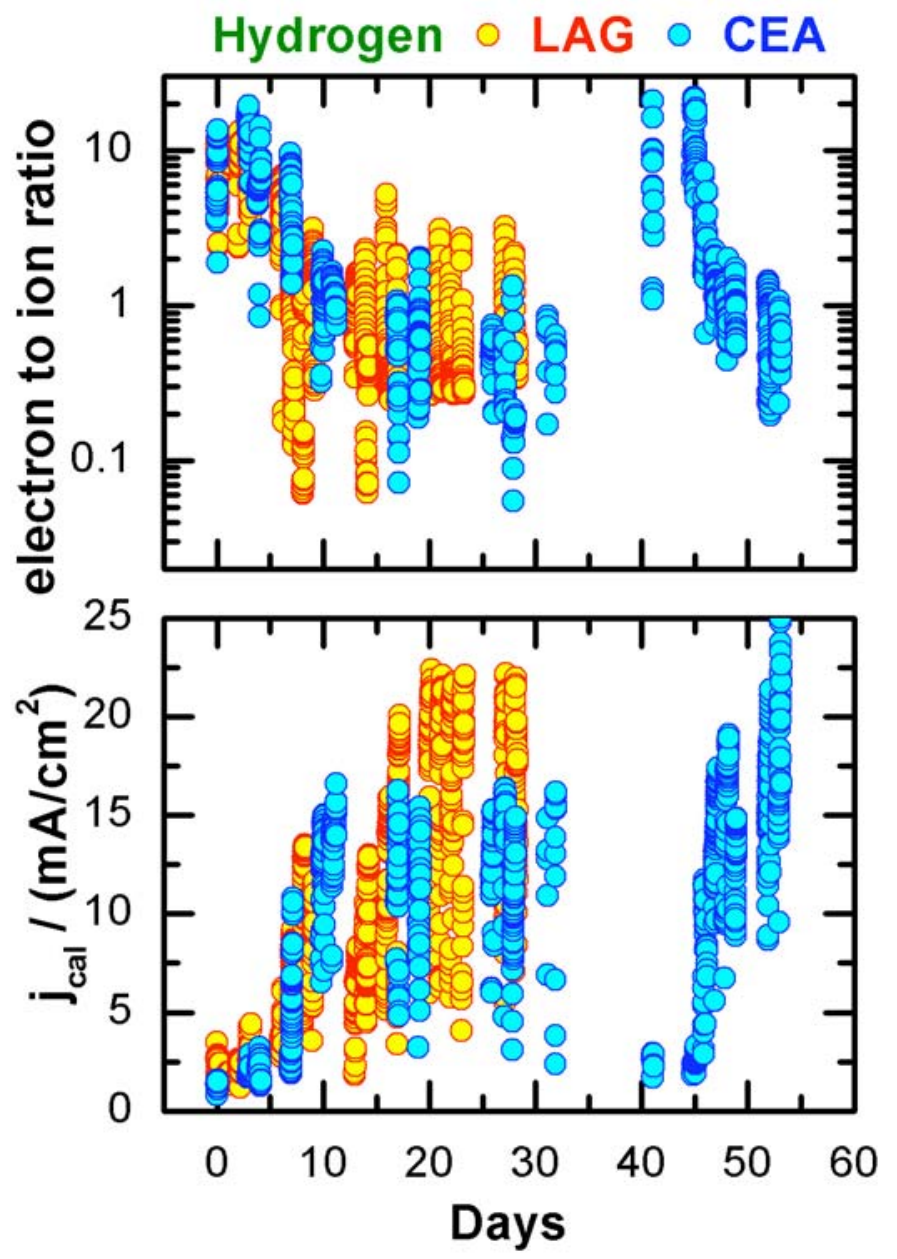

Fig. 2. Comparison of the performance for two grid systems, LAG: $\phi=8 \mathrm{~mm}$, CEA: $\phi=14$ $\mathrm{mm}$. Shown are data obtained during the two experimental campaigns. Obviously, high performance is obtained only after many days of operation. 


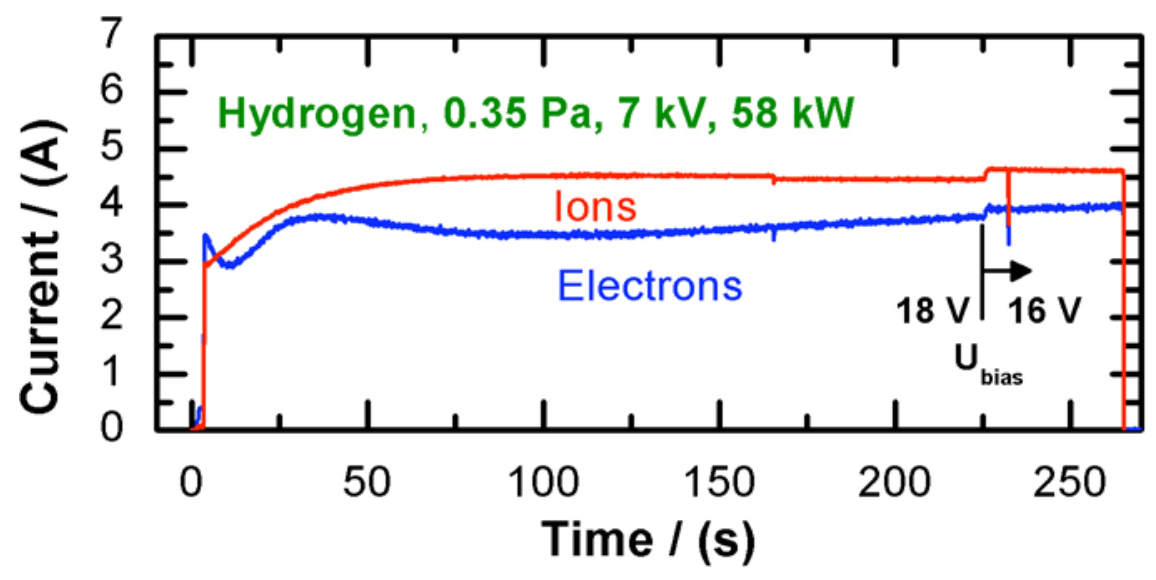

Fig. 3. Extracted ion and electron currents of a long pulse in hydrogen operation. The maximum ion current correspond to $j_{\text {ion }}=230 \mathrm{~A} / \mathrm{m}^{2}$.

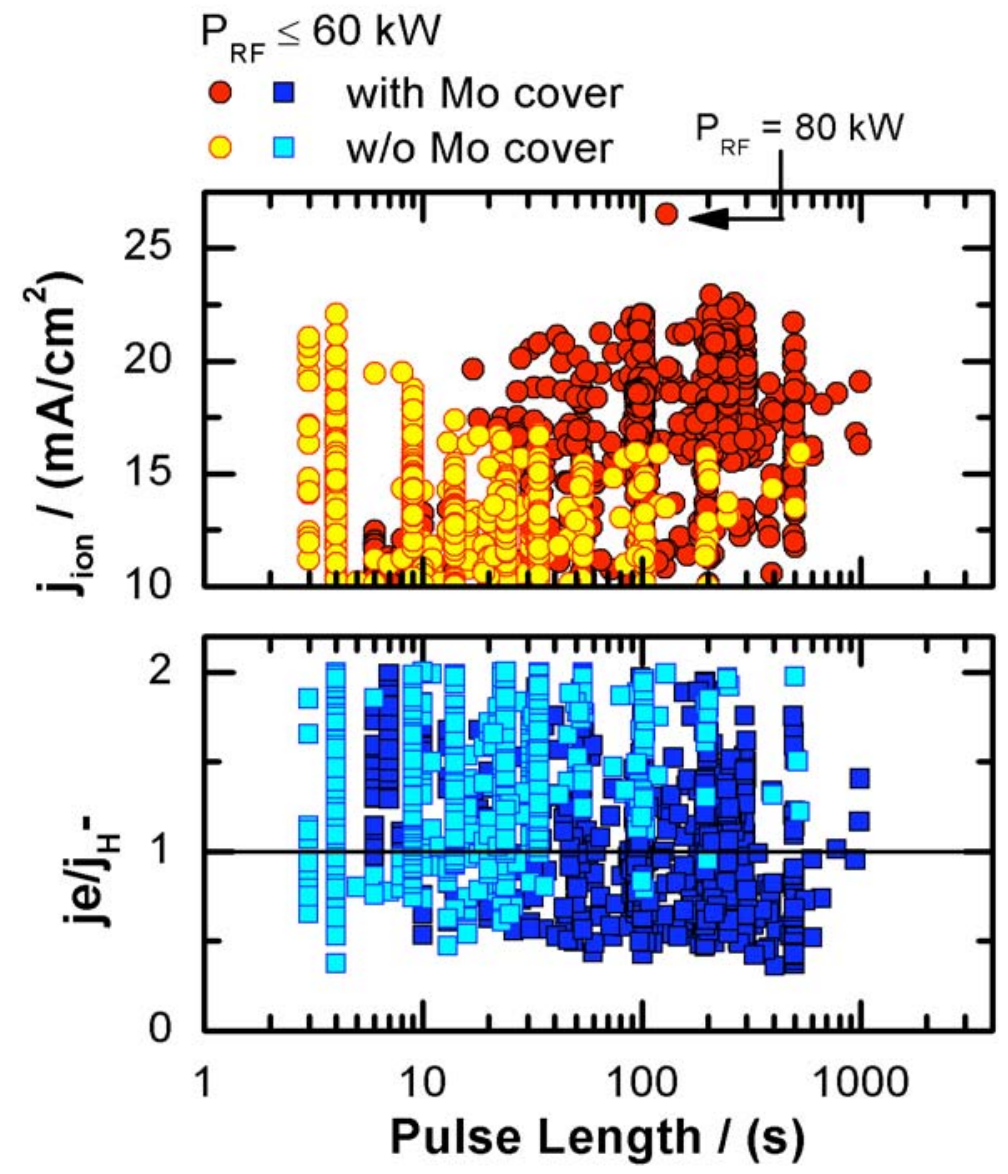

Fig. 4. Performance obtained in MANITU prior to and after Mo-coating. High ion current densities at low electron to ion ratio for long pulses were only obtained after Mo-coating. 


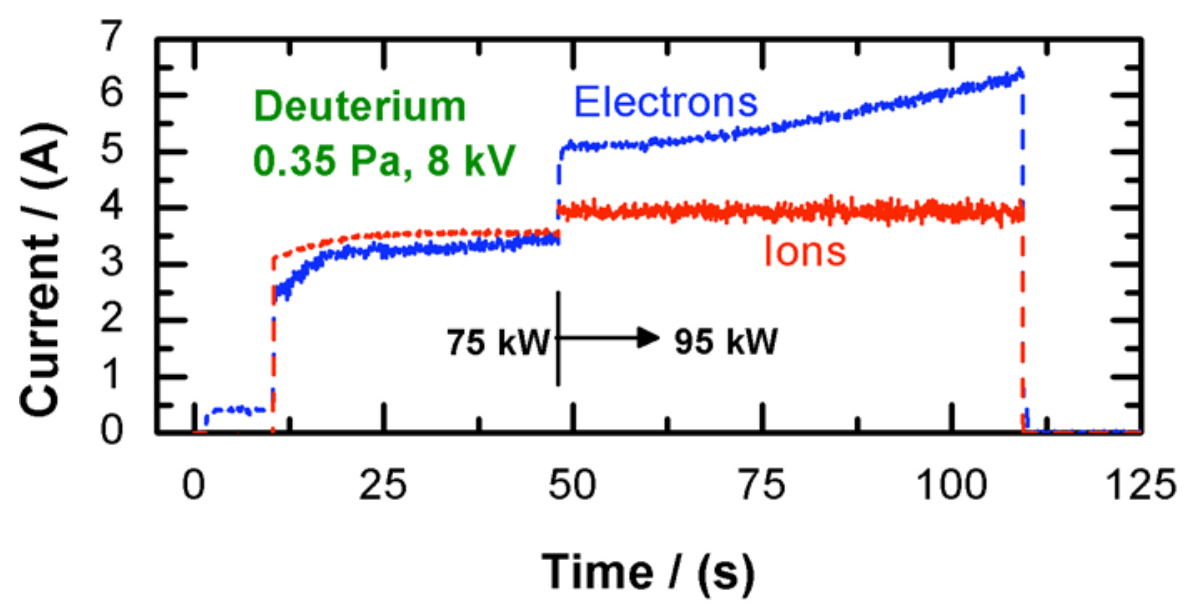

Fig. 5. Extracted ion and electron currents (4 A correspond to $200 \mathrm{~A} / \mathrm{m}^{2}$ ) obtained during a short period of deuterium operation. Due to insufficient Cs conditioning, the electron current becomes high and unstable for the higher RF power.

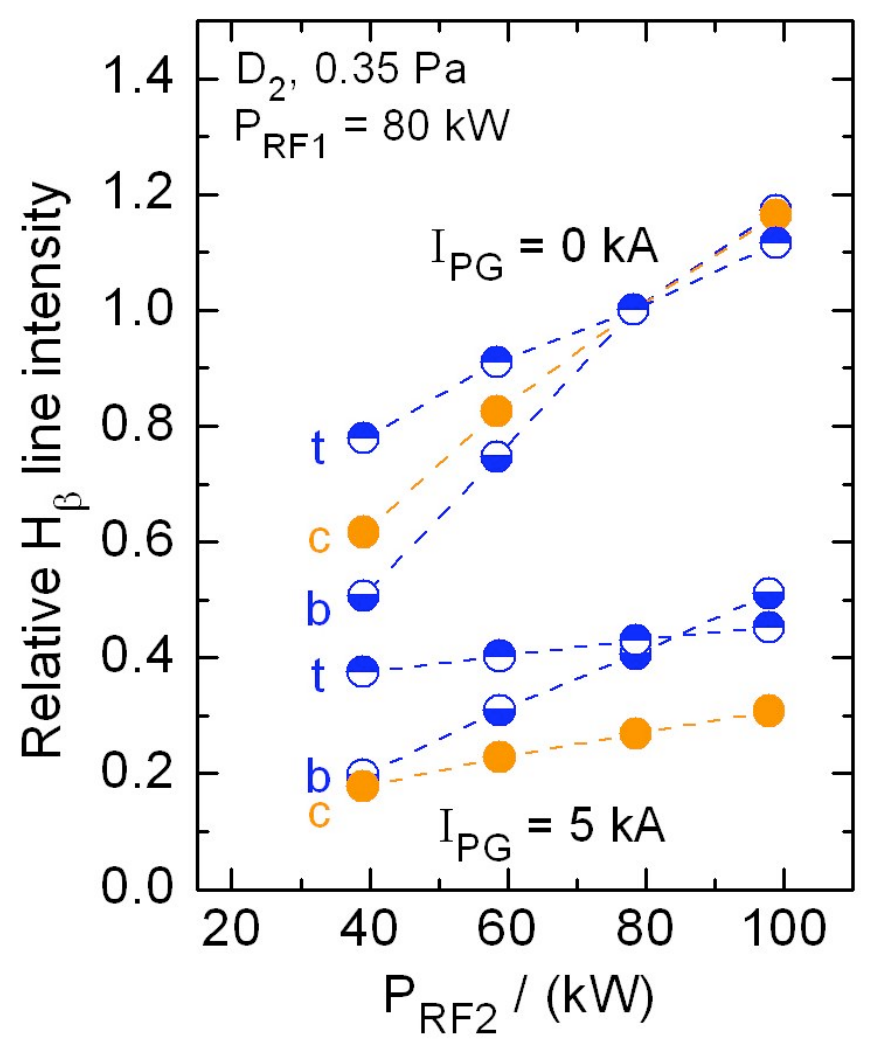

Fig. 6. Line-integrate $\mathrm{H}_{\beta}$-light from horizontal viewing lines close to the extraction region in RADI ( $t$ : line along top part of source, $c$ : central line, $b$ : bottom line). The data were taken without $\left(\mathrm{I}_{\mathrm{PG}}=0\right)$ and with filter field $\left(\mathrm{I}_{\mathrm{PG}}=5 \mathrm{kA}\right)$ for constant $\mathrm{RF}$ power $(80 \mathrm{~kW})$ in the top drivers and a power scan $(40-100 \mathrm{~kW})$ in the bottom drivers. The lower intensity with filter field reflects the lower electron temperature in this case. 